\title{
OCORRENCIA DE INFECÇĀO NATURAL DE FASCIOLA HEPATICA LINNAEUS, 1758 EM LYMNAEA COLUMELLA SAY, 1817 NO VALE DO PARAIBA, SP, BRASIL
}

\author{
Marlene Tiduko Ueta*
}

\begin{abstract}
RSPUB9/504
Ueta, M. T. Ocorrência de infecção natural de Fasciola hepatica Linnaeus, 1758 em Lymnaea columella Say, 1817 no Vale do Paraiba, SP, Brasil. Rev. Saúde públ., S. Paulo, 14:230-3, 1980.

Resumo: Foram registradas em Piquete, no vale do rio Paraiba do Sul (SP), Brasil, taxas de 1,22\% e 0,14\% de infeç̧âo natural em Lymnaea columella, por Fasciola hepatica. Em um único exemplar de Lymnaea columella dentre os 1.052 examinados, foram observadas rédias com xifidiocercárias, rédias com cercárias de Fasciola hepatica e metacercárias de Echinostomatidae.

Unitermos: Infeç̧ão. Fasciola hepatica, Piquete, SP, Brasil. Lymnaea columella, Piquete, $S P$, Brasil.
\end{abstract}

\section{INTRODUÇAO}

A existência de fasciolose bovina e humana no Vale do Paraiba tem sido assinalada desde 1967. França ' (1967) encontrou $10 \%$ de bovinos abatidos no matadouro municipal de Taubaté infectados com $F$. hepatica. São autóctones do Vale do $\mathrm{Pa}$ raiba 8 dos 24 casos humanos assinalados até o presente momento no Brasil (Santos e Vieira ${ }^{18}$, 1965/67; Amato Neto e Silva ${ }^{2}$, 1977 e, Amaral e Busetti ${ }^{1}$, 1979). No Estado do Rio de Janeiro, infecções de animais por $F$. hepatica em municípios do Vale do Paraiba foram assinaladas por l.utz 13 (1921), Rezende e col. ${ }^{15}$ (1973) e por Gomes e col.s (1974). Nesse Estado foram também encontrados limneideos infectados.

Schafranski e col.19 (1977), fazendo levantamento de focos de $F$. hepatica no Vale do Paraiba, encontraram, pela primeira vez em São Paulo, exemplares de Lymnaea naturalmente infectados.
Desde a constatação de casos de fasciolose bovina por França? (1967) e humana por Santos e Vieira 18 (1965/67) no Vale do Paraiba, não há registro de ocorrência de infecção natural em moluscos da região, pois apesar de Santos e França ${ }^{17}$ (1970) terem descoberto mais de uma dezena de criadouros de limneideos no Vale do Paraiba, não fizeram referência à ocorrência de moluscos infectados nos locais pesquisados. Assim, o achado de limneideos naturalmente infectados assinalados por nós, na presente publicação, constitui-se no segundo registro desta natureza em São Paulo.

\section{MATERIAL E METODO}

Em pesquisas de caramujos realizadas no Município de Piquete (SP) no Vale do Paraiba, entre junho - julho de 1977 e

* Do Departamento de Parasitologia do Instituto de Biologia da UNICAMP - Caixa Postal 1170 - 13100 - Campinas, SP - Brasil. 
UETA, M. T. Ocorrência de infeç̧ão natural de Fasciola hspatica, Linnaeus, 1758 em Lymnaea columella Say, 1817 no Vale do Paraíba, SP, Brasil. Rev. Saude públ, S. Paulo, $14: 230-3,1980$.

em setembro de 1978, foram coletados respectivamente 327 e 725 exemplares de Lymnaea columella. Estes exemplares eram trazidos ao laboratório e diariamente expostos à luz artificial, individualmente ou em pequenos grupos. Os espécimes com concha mais clara eram examinados sob microscóp:o estereoscópico para averiguação da presença de parasitas no interior do corpo. No entanto, na maior parte das vezes, somente pudemos constatar presença de infecção por $F$. hepatica ao examinarmos limneas mortas ou moribundas.

\section{RESULTADOS}

Foram encontrados 4 e 1 exemplares infectados por $F$. hepatica, correspondendo a $1,22 \%$ e a $0,14 \%$, respectivamente.

Em laboratório, apenas uma das limneas naturalmente infectadas eliminou cercárias espontaneamente três dıas após a coleta. Esta limnea eliminou, antes de morrer, poucas cercárias que encistaram, mas a maioria dessas formas larvares não chegou a sair das rédias.

Nos outros exemplares de limnea a infecção natural pela $F$. hepatica foi constatada após a morte dos caramujos. Em todas estas limneas mortas foram observadas inúmeras rédias, contendo cercárias, que abarrotavam o hepatopâncreas.

Foi encontrado em um exemplar de $L$. columella a presença concom.tante de rédias de $F$. hepatica e metacercárias de Echinostomatidae.

De 1.052 limneas examinadas, um exemplar apresentou infecção tripla por:

1) rédias contendo cercárias de $F$. hepatica

2) rédias contendo xifidiocercárias, não identificadas, com eliminação espontânea destas cercárias pelo caramujo

3) metacercárias de Echinostomatidae, no teto da cavidade pulmonar, nas proximidades do tubo renal.

\section{DISCUSSAO}

Pequena percentagem de limneideos do Vale do Paraiba, naturalmente infectados por $F$. hepatica, foi encontrada por Lutz ${ }^{13}$ em 1921, ao constatar dois exemplares mortos com rédias e cercárias.

Rezende e col.15 (19'3) encontraram no Rio de Janeiro 26 exemplares de $L$. columella intectados em um total de 1.100 analisado3, o que corresponde a 2,36\%. Este dado, apesar de ser duas vezes maior que o encontrado por nós em 1977, é ainda uma cifra baixa.

Gonzales e col.9 (1974) também assinalaram, no Rio Grande do Sul, o encontro de rédias e cercárıs de $F$. hepatica $\mathrm{cm}$ L. columella coletadas no campo, mas não fizeram referência a percentagtm de infecção natural.

Vários autores relataram infecção natural de $F$. hepatica em outras espécies de Lymnaea, scmpre referindo taxằs baıxas.

Bacigalupo 3,4 (1532) citou o achaco de exemplares de $L$. viatrix naturalmente infectados em Buenos Aires, mas nc̃o registrou a prevalência da infecção entre os caramujos coletados.

Hoffman 10 (1930) relatou que em mais de dois anos de coletas realizadas em várias localidades de Porto Kico encontrou poucos L. cubensis elimiıanco cercárias de $F$. hepatica. Briceño-Rossi ${ }^{6}$ (1950) examinando mais de 400 exemplares de $L$. cuvensis na Venezuela, não encontrou vestígios de larvas de fasciola $\mathrm{cm}$ nenhum caramujo.

Olsen 14 (1944) em seu trabalho sobre bionomia de Stagnicola bulimoides techella disse que esta espécie de limnea pode ser encontrada parasitada por $F$. hepatica durante todo o ano, apesar da percentag $\mathrm{m}$ de infeç̧̃̃o variar conforme a época do ano ou mesmo de um mês para outro. Durante o ano de 1941 , de 16.276 caramujos dissecados, Olsen 14 (1944) encontrou 0,0051\% infectado3. Em um tanque temporário, com as condições ideais para a manutenç:̃o do parasitismo, este mesmo autor encontrou no primeiro exame $2,5 \%$ de infecção e no3 
UETA, M. T. Ocorrência de infeç̧ão natural de Fasciola hepatica, Linnaeus, 1758 em Lymnaea columella Say, 1817 no Vale do Paraiba, SP, Brasil. Rev. Saúde públ., S. Paulo, $14: 230-3, \quad 1980$.

três meses subsequientes $6,6 \% ; 0,32 \%$ e $1,6 \%$, respectivamente.

Em relação a $L$. truncatula naturalmente parasitada por $F$. hepatica, Kendall 11 (1950) verificou uma taxa de infecção entre 5,0 a $6,0 \%$.

Infecções mistas de equinostomas com xifidiocercárias foram assinaladas por Wesenberg-Lund, 1934 (citado por Lie e col.12, 1968) como um dos tipos mais comuns de infecções duplas encontradas em caramujos de campo.

Em relação ao encontro de caramujos com infecção simultânea de Echinostomatidae e $F$. hepatica, Boray ${ }^{5}$ (1967) verificou que $L$. tomentosa era suscetível a $F$. hepatica quando albergava somente metacarcárias de equinostomatideo, mas era refratária quando apresentava rédias e cercárias. Observações de laboratório e de campo feitas por Boray 5 (1967), comprovaram a raridade da infecção mista entre equinostomatideo e $F$. hepatica, uma vez que em 74 exemplares de $L$. tomentosa sujeitas a infecção experimental de ambos os trematódeos, apenas duas mostraram infeç̧ão simultânea. Verificou também que de 6.281 exemplares de campo examinados, apenas um era portador de infecção mista.

Infeç̧ões mistas de Fasciola com larvas de outros trematódeos também foram descritas por Roberts ${ }^{16}$ (1950), ao estudar F. hepatica no campo. Nessa ocasião, Roberts encontrou em $L$. truncatula duas formas de cercárias além das de $F$. hepatica, sendo uma furcocercária, não identificada, encontrada em três exemplares de limnea e cercárias do grupo $C$. cambrensis em outros exemplares.

Até o momento não dispomos de nenhuma referência quanto à infecção tripla de $L$. columella por rédias de $F$. hepatica, rédias com xifidiocercárias e metacercárias de Echinostomatidae.

\section{CONCLUSAO}

1) A percentagem de infecção natural de $L$. columella por $F$. hepatica, nas coletas realizadas em Piquete (SP), foi de $1,22 \%$ e $0,14 \%$.

2) Em um exemplar de $L$. columella foram encontradas rédias com cercárias de $F$. hepatica, rédias com xifidiocercárias e metacercárias de Echinostomatidae.

\section{AGRADECIMENTOS}

Aos Drs. Arnor Fadu Saber e Sérgio Vianna e aos funcionários do DIRA de Lorena e Pindamonhangaba, pela colaboração prestada na coleta de moluscos limneideos em Piquete (SP).

RSPUB $9 / 504$

UETA, M. T. [Natural infection by Fasciola hepatica in Lymnaea columella in the Paraiba river valley, S. Paulo, Brazil.J Rev. Saúde públ., S. Paulo, 14: 230-3, 1980.

ABSTRACT: Infection rates of $1.22 \%$ and $0.14 \%$ were obtained in Lymnaea columella snails naturally infected by Fasciola hepatica. Samples of the snails were collected in Piquete, a municipality of Paraiba do Sul, a river valley area in the State of S. Paulo. Also observed was one of the 1052 specimen of the Lymnaea columella rediae which had xiphidiocercariae and rediae with Fasciola hepatica cercariae and metacercariae of Echinostomatidae.

UNITERMS: Infection. Fasciola hepatica, Piquete, SP, Brazil. Lymnaea columella, Piquete, SP, Brazil. 
UETA, M. T. Ocorrência de infecção natural de Fasciola hepatica, Linnaeus, 1758 em Lymnaea columella Say, 1817 no Vale do Paraiba, SP, Brasil. Rev. Saude pübl., S. Paulo, $14: 230-3,1980$.

\section{REFERENCIAS BIBLIOGRAFICAS}

1. AMARAL, A. D. F. \& BUSETTI, E. T. Fasciolose hepática humana no Bras:1. Rev. Inst. Med, trop. S. Paulo, 21: 141-5, 1979.

2. AMATO NETO, V. \& SILVA, L. J. Infecção humana por Frasciola hepatica no Brasil: relato de um novo caso e análise da questão. Rev. Inst. Med. trop. S. Paulo, 19:275-7, 1977.

3. BACIGALUPO, J. Hallazgo en la ciudad de Buenos Aires de Lymnaea viatrix d'Orb., infectada espontaneamente com cercárias de Frasciola hepatica L. Rev. Soc. argent. Biol., 8:511-3, 1932.

4. BACIGALUPO, J. Lymnaea viatrix d'Orb. infectée par des cercaires de Frasciola hepatica, a Buenos Aires, $C$. $R$. Soo. Biol., 111:828, 1932.

5. BORAY, J. C. Host-parasite relationship between lymnaeid snails and Frasciola hepatica. Vet. Med. Rev., p. 132-40, 1967.

6. BRICENO-ROSSI, A, L. Trabajo experimental sobre Frasciola hepatica. Rev. Sanid. Asist. soc., 15:381-8, 1950.

7. FRANÇA, I. Fasciola hepatica em bovinos no Vale do Paraíba, Estado de São Paulo-Brasil. Arq. Esc. Vet., Belo Horizonte, 19:157-9, 1967.

8. GOMES, P. A. C. et al. Infecção experimental de Lymnaea columella Say, 1817, com Fasciola hepatica Linnaeus, 1758, de ocorrência no Estado do Rio de Janeiro. Arq. Univ. Fed. Rur., Rio de Janeiro, 4:35-8, 1974.

9. GONZALES, J. C. et al. Lymnaea columella, hospeleiro intermediário de Fasciola hepatica (Lin 1758) no Rio Grande do Sul, Brasil. Arq. Fac. Vet. UFRGS, Porto Alegre, 2:37-40, 1974.

10. HOFFMAN, W. A. The intermediate host of Fasciola hepatica in Porto Rico. Puerto Rico J. publ. Hlth, 6:89-90, 1930.

11. KENDALL, S. B. Snail hosts of Fasciola hepatica in Britain. J. Helminth., 24 : $63-74,1950$.

12. LIE, K. J. et al. Implications for trematode control of interspecific larval antagonism within snail hosts. Trans. roy. Soc. trop. Med. Hyg., 62:299-319, 1968.

13. LUTZ, A. Sobre a acorrência da Fasciola hepatioa no Estado do Rio de Janeiro. Bol. Inst. Oswaldo Cruz, $\mathrm{R} 10$ de $\mathrm{Ja}$ neiro, 1:9-13, 1 y21.

14. OLSEN, O. W. Bionomics of the lymnaeid snail, Stagn,cola bulinoides techella, the intermediate host of the liver fluke in southern Texas. J. Agric. Kes., 69:389-403, 1944

15. REZENDE, H. E. B. et al. Notas sobre duas espécies de Lymnaea Lamark, 1799, hospedeiros intermediários de Fasciola hepatica L. no Estado do Rio de Janeiro (Moliusca, Gastropoda, Basommatophora, Lymnaeidae), Arq. Unv. Fed. Kur., Rio de Janeiro, 3: 21-3, 1973.

16. ROBERTS, E. W. Studies on the life-cycle of Fasciola hepatica (Linnaeus) and of its snail host, Limnaea (Galba) truncatula (Müller), in the field and under controlled conditions in the laboratory. Ann. trop. Med. Parasit., 44:187-206, 1950.

17. SANTOS, L. \& FRANÇA, I. Descoberta dos primeiros focos dos hospedeiros da Fasciola hepatica no Estado de São Paulo. In: Congresso Brasileiro de Higiene, ${ }^{\circ}{ }^{\circ}$, São Paulo, 1970. Resumo dos trabalhos. São Paulo, 1970. p. 102.

18. SANTOS, L. \& VIEIRA, T. F. Consideraços sobre os sete primeiros casos de fasciolose humana encontrados no Vale do Paraíba, Estado de São Paulo. Rev. Inst. Adolfo Luta, 25,27:95-109, $1965 / 67$.

19. SCHAFRANSKI, N. L. et al. Levantamento de focos de Frasciola hepatica Linnaeus, 1758, no Vale do Parába, Estado de São Paulo: encontro de Lymnaea columslla Say, 1817, naturalmente infectada. [apresentada a 32a. Conferência Anual da Sociєdade Paulista de Medicina Veterinária, 1977].

Recebido para publicą̧̃o em 08/10/1979 Aprovado para publicasáo em 30/10/1979 